\title{
Estudo Prospectivo sobre Processos de Refino de Óleos Comestíveis que Geram Soap Stock como Subproduto
}

\author{
Prospective Study on Edible Oil Refining Processes that Generate Soap \\ Stock as a By-Product
}

\author{
Yara Simone Chaves Sousa ${ }^{1}$ \\ Pamela Dias Rodrigues ${ }^{1}$ \\ Cristina M. Quintella ${ }^{1}$ \\ ${ }^{1}$ Universidade Federal da Bahia, Salvador, BA, Brasil
}

\begin{abstract}
Resumo
Este estudo prospectivo direcionou-se ao mapeamento de tecnologias de refino alcalino de óleos ou de gorduras comestíveis, identificando a evolução anual, a distribuição por países e setores de origem das tecnologias, os principais titulares e inventores e os métodos mais frequentes. Utilizou-se a base de dados do Espacenet combinando inicialmente palavras-chave para encontrar o maior número possível de códigos da Classificação Internacional de Patentes (CIP), dos quais se empregaram A23D e C11B3/06 para compor a estratégia final, sendo analisadas 233 patentes. Aproximadamente, $81,12 \%$ das patentes foram depositadas nos últimos dez anos, $63 \%$ delas só na China. Empresas alimentícias são os titulares mais frequentes em patentes dessa área (79\%), sendo 59\% de indústrias chinesas. Identificou-se que esta é uma tecnologia emergente com vários gargalos ainda pouco explorados.
\end{abstract}

Palavras-chave: Óleos Comestíveis. Refino Alcalino. Soap Stock.

\begin{abstract}
This prospective study aimed at the mapping of refining technologies for edible oils or fats, identifying the annul evolution, distribution by countries and sectors of origino of the technologies, main owners and inventors and the most frequent methods. The Espacenet database was used, initially combining keywords to find the largest possible number of international patent codes (CIP), of which A23D and C11B3/06 were used to compose the final strategy, with 233 patents being analyzed. Approximately $81.12 \%$ of patents have been filed in the past tem years, $63 \%$ in China alone. Food companies are the most frequent holders of patents in this área (79\%), 59\% of which are Chinese industries. It was identified that this is an emerging, with several bottlenecks still little explored.
\end{abstract}

Keywords: Edible Oils. Alkaline Refining. Soap Stock.

Área Tecnológica: Química. Multidisciplinar. 


\section{Introdução}

Os óleos comestíveis são ingredientes substanciais para a dieta humana como fontes de energia, ácidos graxos e vitaminas lipossolúveis, por exemplo, a vitamina A (Adhami et al., 2019). São amplamente utilizados nas indústrias culinárias, de processamento de alimentos, na produção de cosméticos e na ração animal, o que contribuiu para um considerável aumento no consumo mundial desses produtos nos últimos anos. Óleos vegetais e gorduras também são empregados na produção de biocombustíveis, especialmente biodiesel (MELO et al., 2019; HILTEN et al., 2011; RODRIGUES; QUINTELLA, 2017). O óleo de origem vegetal é considerado a opção alimentícia mais saudável por conter maior teor de ácidos graxos insaturados quando comparado às gorduras animais (SHAHBANDEH, 2020).

Os Estados Unidos e a China são as duas maiores economias e onde ainda há grande consumo de óleos e de gorduras comestíveis, porém cenários distintos podem ser percebidos. Si e Scott (2019), em matéria para o Diálogo Chino, apontam que, com o crescimento da economia chinesa, a classe média emergente vem mudando seus hábitos alimentares e, por isso, houve um aumento no consumo de óleos, alimentos de origem animal e açúcar. Consequentemente, o aumento acelerado de doenças como hipertensão arterial, obesidade, diabetes e doenças cardiovasculares tornou-se umas das questões mais preocupantes. Um em cada três adultos são hipertensos, e o país tem uma das maiores populações de diabéticos do mundo (114 milhões), o que gera uma crise na saúde pública capaz de sobrecarregar o desenvolvimento sustentável da China e, por tabela, do mundo inteiro.

Com relação aos Estados Unidos, mundialmente conhecidos por suas redes de fast food, a dieta média de sua população consiste em excesso de sódio, gordura saturada, grãos refinados e calorias de gorduras sólidas e açúcar, de acordo com dados divulgados pelo Dietary Guidelines for Americans. Também consomem menos vegetais, frutas, grãos inteiros, laticínios e óleos do que o recomendado. Aproximadamente, $35 \%$ dos adultos sofrem de obesidade e estima-se que essa estatística aumente para cerca de $50 \%$ em 15 anos. Embora os efeitos deletérios de uma dieta pouco saudável sejam conhecidos, o público em geral estadunidense parece não ter se conscientizado desse problema com toda sua amplitude (WALKER, 2015).

Os óleos vegetais podem ser produzidos a partir das mais variadas fontes de plantas oleaginosas e polpas de frutos, mas as principais matérias-primas utilizadas na produção de óleo comestível são palma, soja, o milho, colza, girassol, algodão, oliva, coco e amendoim (MELO et al., 2019).

Algumas matérias-primas possuem propriedades medicinais conhecidas e podem ser potencialmente exploradas para a produção de óleo refinado voltado para os consumidores que buscam no alimento benefícios auxiliares para o combate ou a prevenção de doenças. A colza, também conhecida como canola, por exemplo, por ser rica em gorduras insaturadas, pode ajudar no controle das taxas de colesterol no sangue, sendo útil para prevenir doenças cardiovasculares (FU et al., 2016). A peônia, espécie de planta nativa da China, possui maior teor de ácido $\square$-linolênico que outras sementes de oleaginosas, como milho, palma, girassol, azeitona, camélia, entre outros, além de outras propriedades que indicam o óleo de peônia como um alimento muito benéfico à saúde (LI et al., 2015).

O óleo bruto contém impurezas, como os ácidos graxos livres, acilgliceróis, fosfatídeos, hidrocarbonetos, pigmentos, fragmentos de proteína, metais pesados, produtos de oxidação, 
entre outros, presentes naturalmente nas matérias-primas ou adquiridas durante a colheita e o armazenamento das sementes e o processo de extração. Os ácidos graxos livres estão presentes em maior quantidade e são mais suscetíveis a sofrer processos de oxidação, o que contribui para a rancidez oxidativa dos óleos comestíveis, alterando sabor, odor e aparência do alimento. Além disso, é amplamente conhecido que os contaminantes químicos provocam efeitos deletérios à saúde humana. Sendo a estabilidade oxidativa um indicador crítico de qualidade e diretamente relacionada à sua vida útil, o óleo bruto precisa ser submetido a uma etapa de refino para remoção de componentes indesejáveis (TAVAKOLI et al., 2019; MELO et al., 2019).

A produção de óleos comestíveis provoca o acúmulo de uma grande quantidade de subprodutos, sendo o soap stock o principal resíduo do processamento desses produtos, obtido por meio da reação de ácidos graxos livres com álcalis, constituindo a etapa de neutralização do processo de refino químico, a qual geralmente é combinada à degomagem por tratamento com ácido fosfórico ou ácido cítrico antes da adição de solução alcalina para que os fosfolipídios sejam removidos (POYARKOVA et al., 2013; DUIJN, 2016).

Nos últimos anos, vem sendo observada uma tendência das indústrias de processamento em produzir bens de alta qualidade com redução do uso de matérias-primas, energia e melhor eficiência do processo, diminuindo, consequentemente, os custos de produção. Diversos fatores podem ter direcionado a esse comportamento, como a inserção de políticas internacionais para redução dos impactos negativos ao meio ambiente e a necessidade de otimizar os custos de produção, visando à permanência em um mercado global cada vez mais competitivo. Também é notável o aumento no interesse por parte dos consumidores de produtos alimentícios em adquirir mercadorias produzidas com responsabilidade ambiental (NUCCI et al., 2014).

Como o soap stock é um subproduto inerente ao processo de refino de óleo comestível, sua disposição no meio ambiente é essencial na operação contínua de uma refinaria, o que provoca impactos ecológicos negativos (ALIPOUR et al., 2017). No passado, resíduos industriais eram tratados apenas para atender às exigências das regulamentações ambientais. Contudo, a abundância de rejeitos industriais e a crescente necessidade de uma sociedade com práticas cada vez mais sustentáveis impulsionaram a busca por tecnologias para transformar resíduos em produtos úteis, ao invés de simplesmente submetê-los a tratamento antes de serem descarregados no meio ambiente (LEE et al., 2019).

O soap stock (borra de refino) é constituído de água, ácidos graxos livres, óleo neutro, fosfatos hidrolisados, matéria insaponificável, proteínas e substâncias mucilaginosas (ricas em polissacarídeos). Embora seja considerado um resíduo de baixo valor econômico, o soap stock constitui uma valiosa fonte de ácidos graxos que tem ampla aplicação industrial, como obtenção de produtos químicos derivados, produção de surfactantes, detergentes, sabões, óleos, lubrificantes e biodiesel (FAZLI et al., 2013; PANTOJA et al., 2019). O soap stock também é empregado como ingrediente em ração animal, aditivos tecnológicos para borrachas, em indústria cosmética, emulsificação, lavagem, dispersão, plastificação, estruturação e modificação de propriedades reológicas de alguns sistemas (POYARKOVA et al., 2013; POYARKOVA et al., 2012).

A propriedade surfactante do soap stock é particularmente interessante para aplicação em recuperação avançada de petróleo, pois a injeção de surfactantes é considerada uma das estratégias mais promissoras para aumentar a produção de petróleo após a utilização de métodos convencionais, podendo alterar a molhabilidade da rocha do reservatório e diminuir a tensão 
interfacial entre óleo e água, aumentando significativamente a recuperação final de petróleo (NI et al., 2020; MARQUES et al., 2014).

$\mathrm{Na}$ literatura há poucos registros de uso de soap stock para recuperação avançada de petróleo. Barbosa et al. (2009) utilizaram soap stock na composição de fluidos de recuperação avançada e obtiveram um aumento de cerca de 5 a $15 \%$ na recuperação do óleo.

O presente trabalho tem como objetivo mapear as tecnologias apropriadas de refino de óleos ou de gorduras comestíveis por reação química com bases que geram soap stock como subproduto, identificando a evolução anual, a distribuição por países e setores de origem das tecnologias, os principais titulares e inventores e os métodos mais frequentes.

\section{Metodologia}

Para mapear os documentos de patentes referentes às tecnologias empregadas no refino alcalino de óleo comestível, utilizou-se a base de dados on-line European Patent Office (EPO) com acesso livre para cerca de 120 milhões de documentos de patentes. Os dados apresentados foram baixados em março de 2020.

A Tabela 1 apresenta o escopo com os códigos e as palavras-chave empregados para a busca inicial e, em destaque, a combinação de códigos utilizada como estratégia final para a varredura de documentos.

Tabela 1 - Estratégia de busca

\begin{tabular}{|c|c|c|c|c|c|c|c|c|}
\hline $\begin{array}{c}\text { Códigos E } \\
\text { PALAVRAS-CHAVE }\end{array}$ & \multicolumn{8}{|c|}{ EstratÉGIA DE BUSCA } \\
\hline $\begin{array}{c}\text { Soap stock* or soapstock* } \\
\text { and oil refining* }\end{array}$ & $\mathrm{x}$ & & & & & & & \\
\hline A23D & & $\mathrm{x}$ & & & & & & \\
\hline A23D9/00 & & & $\mathrm{x}$ & & & & & \\
\hline A23D9/02 & & & & $\mathrm{x}$ & & & & \\
\hline A23L33/115 & & & & & $\mathrm{x}$ & & & \\
\hline $\mathrm{C} 11 \mathrm{~B} 3 / 00$ & & & & & & $\mathrm{x}$ & & \\
\hline $\mathrm{C} 11 \mathrm{~B} 3 / 06$ & & & & & & & $\mathrm{x}$ & \\
\hline $\mathrm{A} 23 \mathrm{D}$ and $\mathrm{C} 11 \mathrm{~B} 3 / 06$ & & & & & & & & $\mathrm{x}$ \\
\hline Número total de patentes & 103 & $>10.000$ & $>10.000$ & 5.667 & 3.500 & 6.265 & 2.147 & 426 \\
\hline Famílias & 1,1 & 1,0 & 1,0 & 1,0 & 1,0 & 1,0 & 1,1 & 1,8 \\
\hline & & tal de pate & ntes proce & adas & & & & 233 \\
\hline
\end{tabular}

Fonte: Elaborada pelas autoras deste artigo (2020)

Inicialmente foram combinadas palavras-chave associados ao tema, empregando-as com os operadores lógicos "or" e "and" para encontrar o maior número de códigos de Classificação Internacional de Patentes (CIP) e os mais relevantes encontrados nessa busca foram utilizados para alcançar a estratégia final que consistiu na associação dos códigos A23D e C11B3/06 de modo a abranger o maior número possível de patentes de forma seleta para o assunto de 
interesse. Essa metodologia foi baseada nas mesmas adotadas em estudos anteriores (SAMPAIO et al., 2019; SANTOS et al., 2018; RODRIGUES; QUINTELLA, 2017). Os códigos selecionados referem-se especificamente a: (i) A23D, óleos ou gorduras comestíveis, por exemplo, margarinas, gorduras para bolo, óleo para cozinhar (extração, refinação, conservação, hidrogenação); e (ii) C11B3/06, refinação de gorduras ou óleos graxos por reação química com bases. Os demais códigos da CIP utilizados no escopo referem-se especificamente a: (i) A23D9/00, outros óleos ou gorduras comestíveis, por exemplo, gorduras para bolo, óleo para cozinhar; (ii) A23D9/02, produção ou preparação de outros óleos ou gorduras comestíveis, por exemplo, gorduras para bolo, óleo para cozinhar; (iii) A23L33/115, preparo ou tratamento de ácidos graxos ou seus derivados; preparo ou tratamento de gorduras ou óleos; e (iv) C11B1/00, produção de gorduras ou óleos graxos a partir de matérias-primas.

Foram encontrados 426 documentos de patentes sobre tecnologias de refino alcalino de óleo comestível. Contudo, fez-se necessária a remoção de 193 patentes replicadas, totalizando 233 registros restantes.

\section{Resultados e Discussão}

A Figura 1 apresenta a evolução anual no depósito de patentes, na qual se observa um aumento significativo a partir de 2010. O primeiro documento de patente sobre refino de óleo comestível foi requerido em 1982. Os inventores Traitler e Winter forneceram um método de preparação de óleo refinado do fruto do gênero Ribes, com baixo teor de ácidos graxos livres, rico em ácido gama-linolênico que é benéfico à saúde humana e de baixo custo. Em seu método, o óleo bruto é extraído a partir de resíduos secos e moídos da produção de sucos, geleias, conhaques, licores e aguardentes da fruta (TRAITLER; WINTER, 1982).

Figura 1 - Evolução anual de patentes

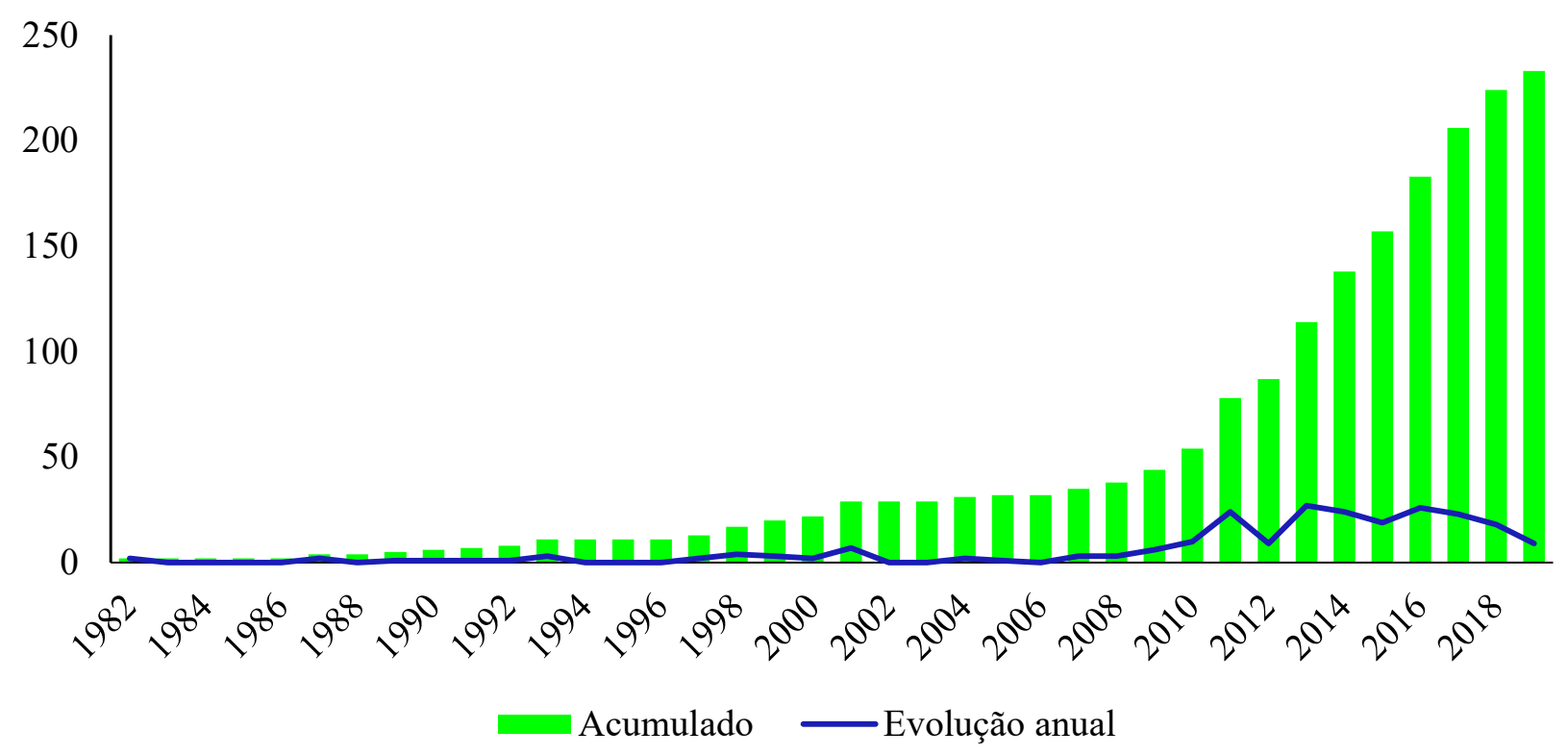

Fonte: Elaborada pelas autoras deste artigo (2020) 
No período de 2010 até 2019, foram depositadas 189 patentes, representando 81,12\% do total de patentes requeridas ao longo de quase 40 anos, evidenciando que essa tecnologia é ainda emergente. É possível observar que o auge de depósitos ocorreu entre 2013 e 2016 (26 patentes). Também é notório que houve um esforço das entidades requerentes, sejam elas indústrias, instituições de pesquisa ou independentes, para alcançar melhorias nas técnicas de produção de óleo comestível refinado. Relacionando o aumento no consumo mundial de óleos comestíveis às tendências de mercado já citadas, espera-se que maiores esforços sejam concentrados, gerando aumento no número de divulgações tecnológicas.

Diversas patentes analisadas se referiram a processos para obter produtos de alta qualidade, com menor custo e redução de poluentes. Sandberg e Thurstad (2012), por exemplo, divulgaram um processo para remover de forma eficaz substâncias indesejáveis em uma composição oleosa, obtendo um concentrado altamente purificado enriquecido em ácidos graxos poli-insaturados ômega-3 (SANDBERG; THUSTAD, 2012). Hanbei (2019) patenteou um método de produção de óleo refinado contendo misturas de óleos vegetais de modo a obter um produto final com efeito medicinal capaz de prevenir pressão alta, câncer, derrame, infarto do miocárdio, doenças cardíacas, de auxiliar na perda de peso, de limpar substâncias nocivas no sangue, de aliviar a síndrome da menopausa, de refrescar e proteger o cérebro, de melhorar a atenção e a memória e de proteger o fígado e o estômago (HANBEI, 2019). Song (2019) forneceu um método de produção de óleo refinado rico em ácidos graxos insaturados utilizando um aparato com uma estrutura que combina aquecimento duplo com uma camada de isolamento externa para o tanque de refino alcalino, contribuindo na redução dos custos de produção e de operação, na melhoria da eficiência do processo e na remoção eficaz de impurezas (SONG, 2019).

A Figura 2 apresenta os países de primeira prioridade na área de refino de óleos comestíveis. A China merece maior destaque no desenvolvimento dessa tecnologia por registrar 147 documentos de patente, o equivalente a $63 \%$ do total de patentes processadas. Também há contribuições registradas em outros países, como Japão e Estados Unidos com $13 \%$ e 7\%, respectivamente. Além dessas, algumas patentes foram depositadas em protocolo sem indicação de país, seis pela Organização Mundial da Propriedade Intelectual (OMPI) e quatro patentes pela Organização Europeia de Patentes (OEP). Apenas quatro patentes foram depositadas no Brasil como país de primeira prioridade.

Os Estados Unidos, a China e o Japão são as três maiores economias do mundo e é previsível que sejam os maiores depositantes de patentes sobre refino de óleo comestível. De acordo com os dados, a China é a área legal em que mais ocorrem pedidos de patentes sobre métodos de refino de óleo comestível, sobretudo nos últimos dez anos. Esse panorama não surpreende se for considerado o aumento do consumo de óleos comestíveis nos hábitos alimentares da população chinesa, o que gerou preocupação com uma possível crise na saúde pública, como já foi mencionado (SI; SCOTT, 2019). 
Figura 2 - Principais países com maior número de depósitos de patentes na área de refino de óleo comestível

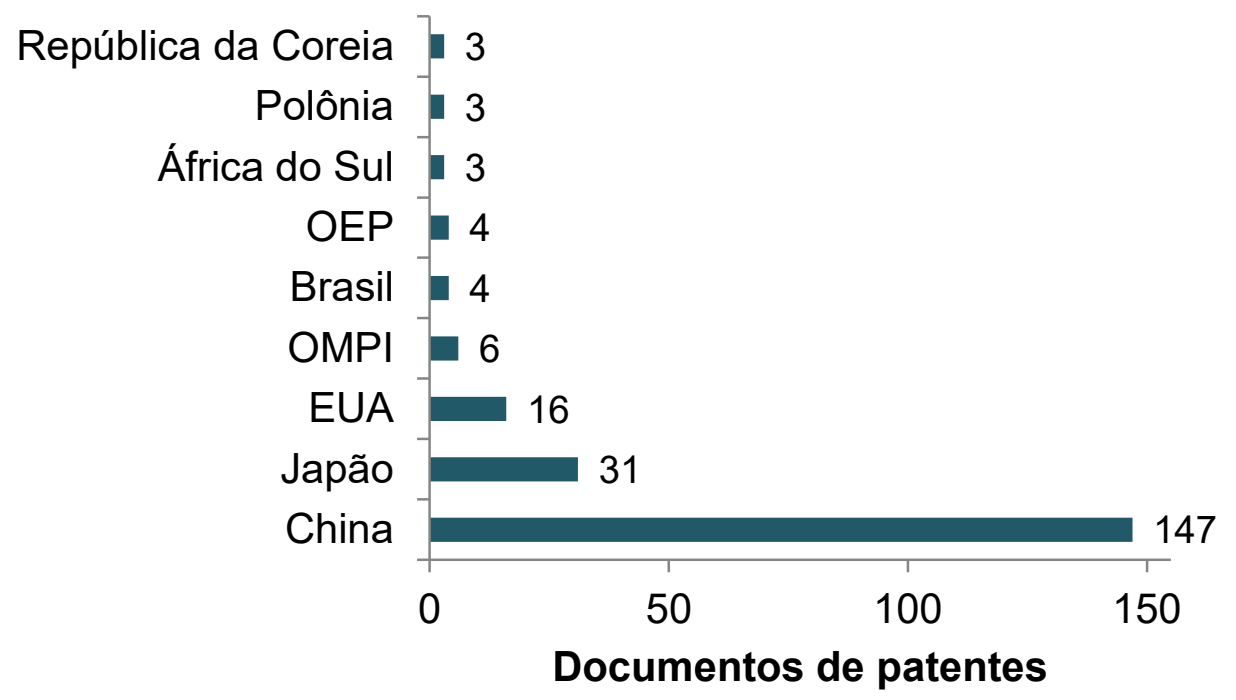

Nota: Siglas: OEP - Organização Europeia de Patentes; OMPI - Organização Mundial da Propriedade Intelectual

Fonte: Elaborada pelas autoras deste artigo (2020)

Uma das mais recentes patentes depositadas na China refere-se a um método para preparação de óleo refinado de colza, ou canola, com melhorias na etapa de degomagem, otimizando o tempo e a eficiência de processamento e preservando o sabor e os nutrientes do produto final, mantendo, assim, a propriedade preventiva contra doenças cardiovasculares (ZIKUAN et al., 2019). Já Zeyang (2016) patenteou uma tecnologia de preparo de óleo de semente de peônia com propriedades medicinais e apresentando melhorias no processo de extração para aprimorar a qualidade do óleo. Ambos os trabalhos evidenciam um interesse por utilização de matérias-primas que possam agregar ao alimento um valor benéfico à saúde.

Com relação às patentes requeridas nos Estados Unidos, nota-se apenas uma tímida contribuição para o aprimoramento de tecnologias de refino de óleo comestível, contabilizando 16 patentes ao longo de quase 40 anos. Admitindo-se os fatores já enumerados que estão impulsionando as indústrias de processamento a direcionarem suas prioridades para aumentar a qualidade dos produtos e reduzir o uso de matérias-primas, energia e custo operacional e sendo essa uma tendência global, presume-se que as indústrias de processamento de alimentos terão que seguir o mesmo comportamento. Com isso, mais contribuições tecnológicas sobre refino de óleos comestíveis podem ser esperadas em todas as partes do mundo (NUCCI et al., 2014).

A Figura 3 refere-se aos tipos de requerentes que depositaram patentes na área de estudo. O setor empresarial foi o de maior destaque, detendo 183 registros de patentes no período, alcançando $79 \%$ das patentes sobre tecnologias de obtenção de óleos comestíveis refinados. As indústrias chinesas são as principais detentoras de patentes, representando $59 \%$ das empresas requerentes. 
Figura 3 - Tipos de requerentes de documentos de patentes
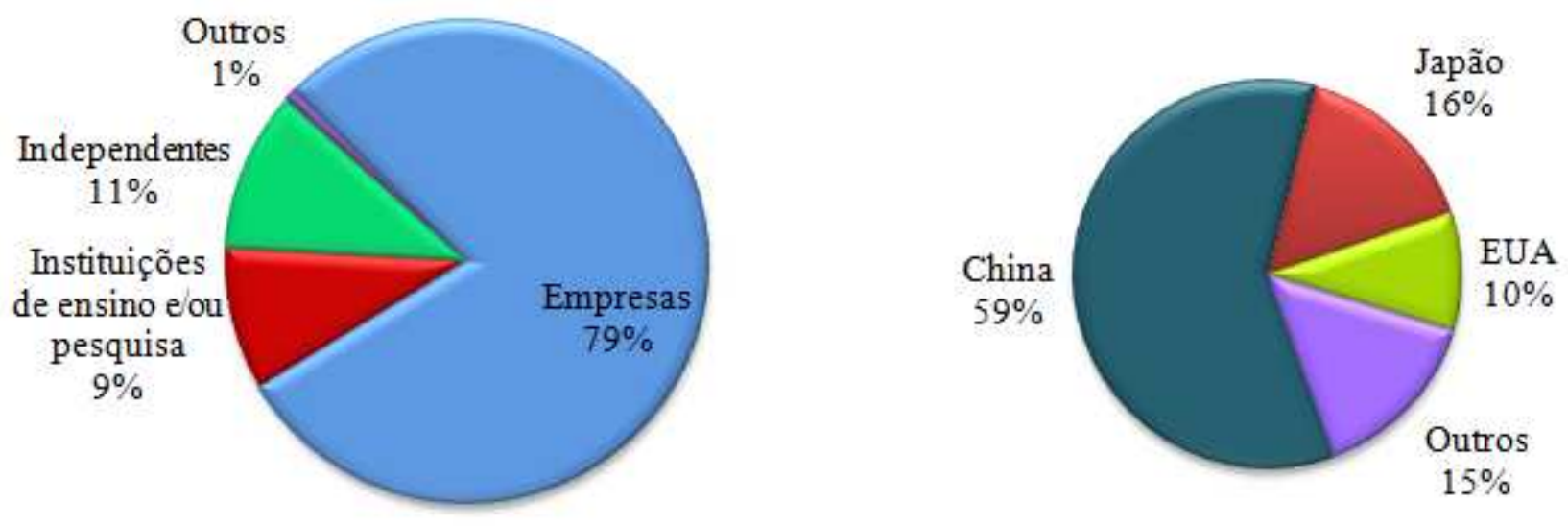

Fonte: Elaborada pelas autoras deste artigo (2020)

Já era esperado que indústrias estivessem à frente de investimentos em pesquisas de desenvolvimento tecnológico, seja com parcerias com centros de pesquisa ou independentes, sobretudo pela necessidade de resolver problemas relacionados à produtividade e aos prejuízos ao meio ambiente, conforme já explanado neste estudo (NUCCI et al., 2014).

As instituições de ensino e/ou pesquisa somaram menos registros, apenas $9 \%$, que os inventores independentes, com $11 \%$. Foram identificadas uma patente em que o depositante é uma entidade sem fins lucrativos (SUN et al., 2013) e outra em que o autor e depositante renunciou ao direito de ser citado, desse modo, elas foram contabilizadas na categoria Outros.

O inventor Hanbei (2019) cita a China como um grande consumidor de óleo comestível, tendo atingido cerca de 38 milhões de toneladas em 2018, sendo os mais tradicionais os óleos de soja, colza, milho, amendoim, girassol e palma, os quais contêm principalmente ácido linoleico, um dos ácidos graxos essenciais para o corpo humano. Na descrição de seu invento, Hanbei cita a inadequação dos teores de nutrientes essenciais, como o ácido linoleico e o ácido linolênico, nos óleos comuns disponíveis no mercado, aos valores recomendados pela Organização Mundial de Saúde (OMS), resultando em deficiências desses nutrientes na população chinesa (HANBEI, 2019).

A Figura 4 A apresenta as principais empresas depositantes de patentes na área de refino de óleo comestível. O grupo Nisshin Oillio é o que mais se destaca no segmento, possuindo nove registros de patentes sobre tecnologias de produção de óleo comestível refinado. Constitui um grupo de empresas japonesas que atuam na fabricação, no processamento e na distribuição de óleos comestíveis e operam em diversos segmentos como óleos e gorduras, gorduras modificadas, Química Fina, alimentos saudáveis, entre outros (NIKKEI ASIAN REVIEW, 2020). Suas patentes referem-se a: métodos de produção de óleo comestível com qualidade melhorada, incluindo características como sabor, odor, cor (MASAYUKI, 2017; MASAYUKI; HIROYUKI, 2017; IWASAWA et al., 2009); métodos de preparação de óleos e gorduras refinadas com teor de ácido oleanólico e ácido maslínico fisiologicamente adequados (KUNO; SHINOHARA, 2001) ou outros componentes desejáveis como os ácidos graxos poli-insaturados (YAMAUCHI et al., 
2001); métodos de produção de óleos e gorduras comestíveis refinados com teor reduzidos de 3-MCPD (3-monocloropropano-1,2-diol) e seus derivados (HIROSHI, 2014; 2015) ou ainda que métodos de produção de óleos vegetais que alie melhorias na qualidade, rendimento do processo, remoção de contaminantes e concentração de componentes úteis como o orizanol, tocoferóis, entre outros (MAKI et al., 2008; HIDAKA; TSUCHIYA, 1998).

A Cargill é uma multinacional americana que fornece produtos e serviços alimentícios, agrícolas, financeiros e industriais ao mundo todo (CARGILL, 2020). Suas patentes referem-se a métodos de preparação de óleo vegetal com teor reduzido de contaminantes, como cloropropanóis, ésteres de ácidos graxos e derivados (FALK, 2018a; 2018b; KRUIDENBERG; FALK, 2011) e métodos de produção de composições de óleos contendo triacilgliceróis (LIU; LAMPERT, 1997; FREEMAN et al., 2001).

A Kao Corporation é uma empresa japonesa que atua no setor de fabricação de produtos químicos, cosméticos, produtos de limpeza, óleos comestíveis, entre outros (KAO, 2020). Suas patentes referem-se a métodos de produção de óleo comestível com baixo teor de contaminantes, como 3-MCPDE e ácidos graxos livres (FUKUHARA et al., 2013) a baixo custo (YOSHINORI; TOSHITERU, 2004), com qualidade melhorada e redução de emissão de poluentes atmosféricos (MINORU; TOSHITERU, 2011) e método de produção de óleo e gordura contendo álcoois triterpenos (TETSUYA et al., 2013).

Figura 4 - A: Principais instituições requerentes na área de refino de óleo comestível; B: Principais inventores

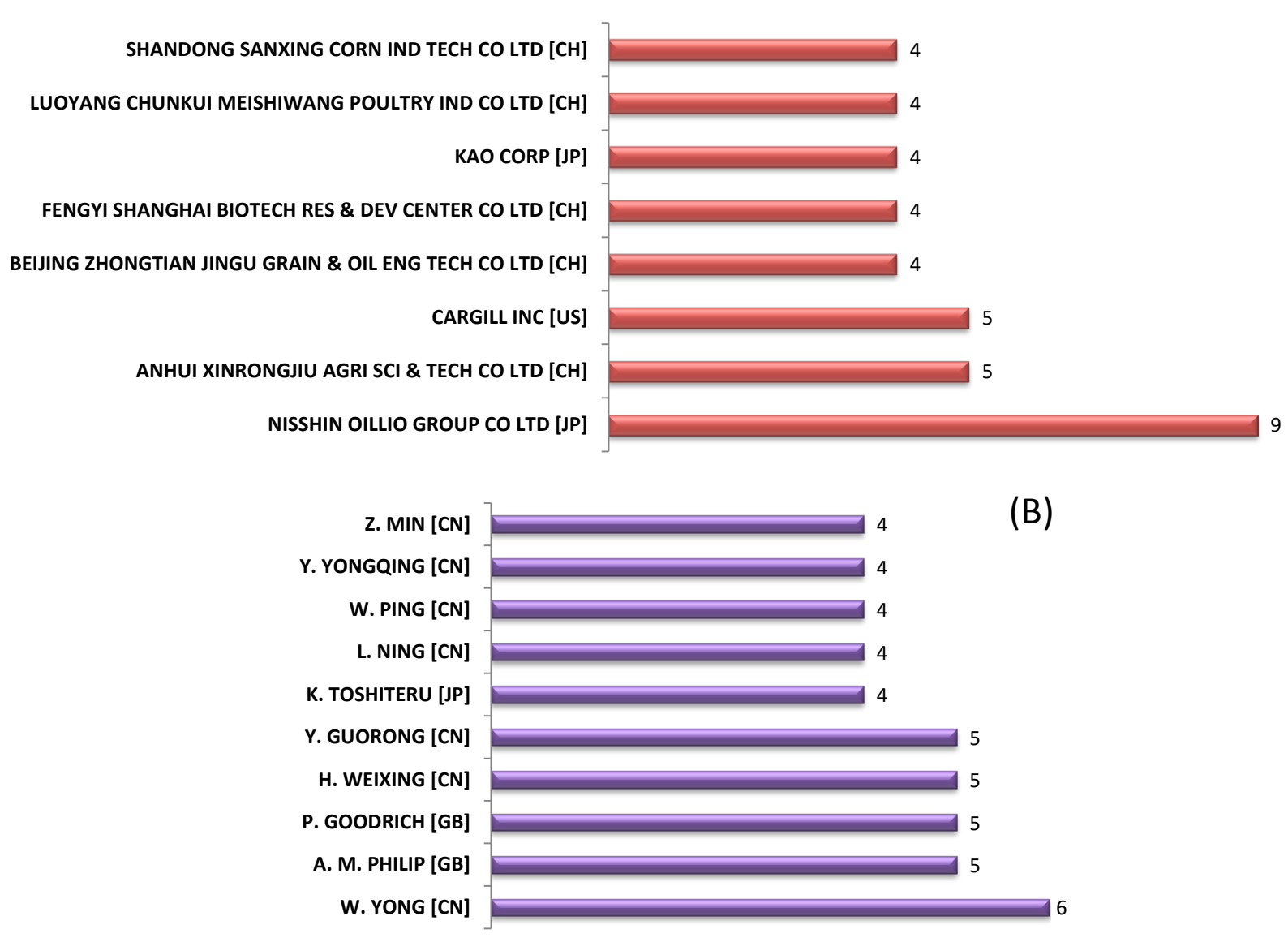

Fonte: Elaborada pelas autoras deste artigo (2020) 
A Figura $4 \mathrm{~B}$ apresenta os inventores que acumulam o maior número de registros de patentes. O chinês Wang Yong é o que mais se destaca, somando seis depósitos de patentes na área de refino de óleo comestível. Suas patentes fornecem métodos de preparação de óleo de origem vegetal ou animal ricos em componentes nutricionais (WANG; ZHOU, 2016; RAMAN, 2011), métodos de preparação de óleos comestíveis com remoção ou menor teor de componentes indesejados (SONG; FANG, 2017; FUKUHARA et al., 2013; SUN et al., 2013) e método de preparação de óleo vegetal com menor custo, menos poluente e melhor qualidade (LIU et al., 2014).

Observa-se uma predominância de inventores chineses entre os principais depositantes. A China, como já mostrado em resultados anteriores, é o país com maior número de depósitos de patentes e também o país em que as empresas mais investem nesse tipo de tecnologia. Destacam-se ainda os inventores britânicos Peter Goodrich e Martin Philip Atkins, cada um com cinco depósitos de patentes sobre tecnologias de produção de óleos refinados.

A Figura 5 apresenta as reivindicações das patentes e as novidades tecnológicas descritas para os métodos de produção. Entre os 233 documentos analisados, 62\% (145) descrevem métodos de produção, ou seja, tecnologias envolvendo etapas de extração do óleo bruto e etapas de refino. As patentes que se referem exclusivamente a etapas de refino equivalem a $29 \%$ (67) dos documentos estudados, enquanto as invenções que fornecem métodos enfatizando o desenvolvimento de aparatos para a produção de óleo refinado representam 9\% (20). Nota-se o enfoque dos inventores pelas tecnologias de produção (extração e refino), que, uma vez mais abrangente, comporta mais possibilidades para inovações.

Figura 5 - A: Reivindicações das patentes; B: Novidades tecnológicas descritas nas patentes dos métodos de produção
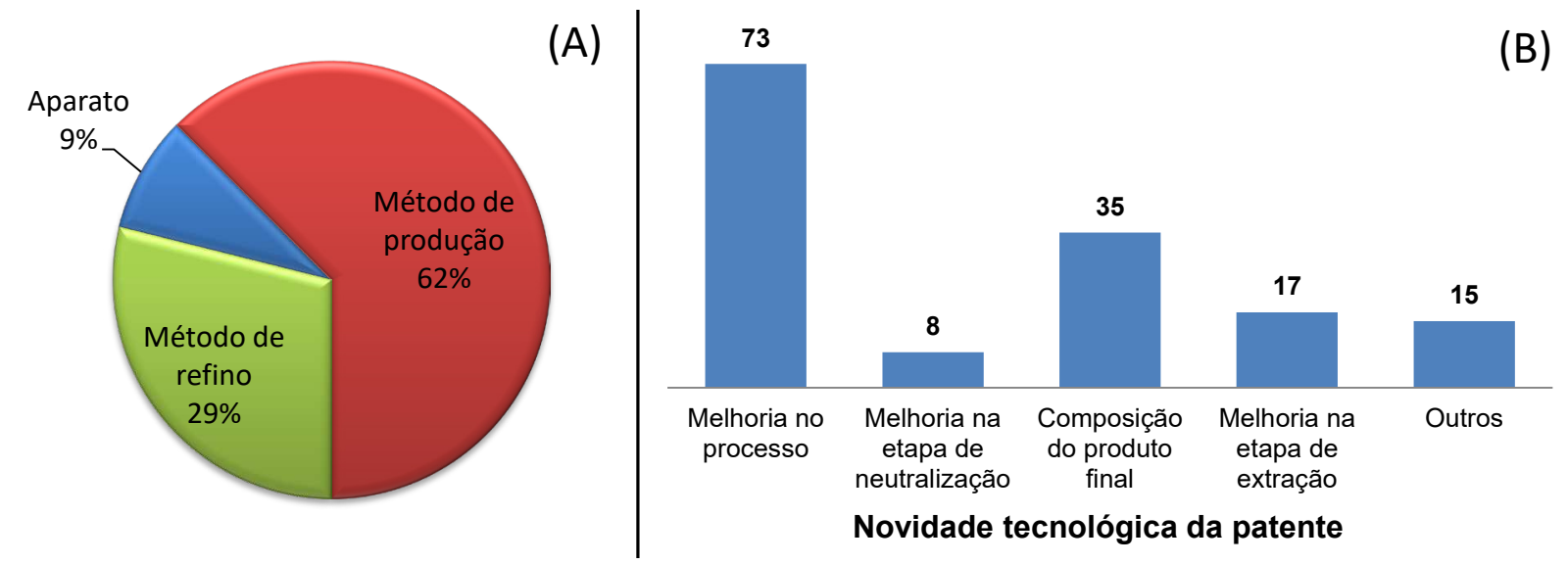

Fonte: Elaborada pelas autoras deste artigo (2020)

Das 145 patentes que descrevem tecnologias de produção, 73 (49\%) destacaram melhorias envolvendo todo o processo e 35 (24\%) enfatizaram como principal vantagem do método a composição alcançada, seja pelo valor nutritivo, pela formulação inédita apropriada para consumo alimentar ou pela aquisição de propriedades biológicas para cuidados com a saúde. Entre as patentes processadas, também foram observadas aquelas que apontaram como principal destaque as melhorias alcançadas na etapa de extração, somando $12 \%$ dos registros referentes à produção. As $10 \%$ remanescentes abordaram métodos com melhorias em outras etapas do processo. Observa-se, a partir de tais resultados, que, nesse cenário tecnológico, maiores es- 
forços foram dedicados para se obter melhorias na eficiência do processo, de modo a reduzir custos de operação, uso de matérias-primas e geração de resíduos sem perder a qualidade do óleo refinado. Nucci et al. (2014) apontaram que, nos últimos anos, há uma tendência mundial de as indústrias de processamento reduzirem custos de produção, utilizarem menos recursos $e$ adotarem mecanismos mais sustentáveis para se manter em um mercado cada vez mais competitivo e ainda atender às políticas internacionais de preservação do meio ambiente.

\section{Considerações Finais}

A tecnologia de refino alcalino de óleo comestível apresenta-se como emergente, sendo encontrado um crescimento significativo somente nos últimos 10 anos, em que foram depositadas 189 patentes que correspondem a $81 \%$ do total de 233 patentes depositadas ao longo de quase 40 anos de desenvolvimento da tecnologia. Relacionando esses dados com informações abordadas neste estudo, espera-se um crescimento no número de contribuições para o cenário tecnológico nos próximos anos.

Observa-se um domínio do desenvolvimento da tecnologia por parte da China que possui prioridade em $63 \%$ das patentes. Esse panorama não surpreendeu, uma vez que, como já reportado na literatura, houve aumento considerável do consumo de óleos comestíveis pelos chineses. Com relação às patentes requeridas nos Estados Unidos, percebeu-se apenas uma tímida contribuição para o aprimoramento tecnológico do refino químico de óleos, contabilizando-se 16 patentes ao longo de quase 40 anos. Admitindo que a prioridade industrial atualmente é a obtenção de produtos de alta qualidade com redução no consumo de matérias-primas, energia e custos operacionais e esta se apresenta como uma tendência mundial, presume-se que as indústrias de processamento de alimentos terão que seguir o mesmo comportamento. Dessa forma, infere-se que mais inovações tecnológicas serão divulgadas em todas as partes do mundo.

O setor empresarial é responsável por $79 \%$ (183 patentes) dos registros de patentes sobre refino de óleo comestível, com destaque para empresas japonesas e chinesas do setor alimentício. O grupo japonês Nisshin Oillio aparece como maior investidor, e suas patentes reivindicam principalmente melhorias na qualidade nutricional do produto final. Destaca-se ainda a multinacional americana Cargill com cinco patentes que reivindicam novos métodos que geram óleos com menor teor de contaminantes ou maior taxa de triacilgliceróis.

Os inventores chineses W. Yong, H. Weixing e Y. Guorong se destacam entre os principais inventores, resultado este que acompanha o forte domínio do desenvolvimento da tecnologia por parte da China.

Foi identificado que as patentes trazem inovações reivindicadas em novos aparatos para o refino, novos métodos de refino do óleo, mas principalmente melhorias no processo de produção do óleo refinado. Entre as patentes de métodos de produção, destacam-se principalmente as novidades tecnológicas que levam a melhorias no processo de produção como um todo, mas também agregam valor nutricional na composição do produto final e várias delas com propriedades medicinais. 


\section{Perspectivas Futuras}

O estudo prospectivo sobre refino de óleo comestível demonstrou que essa é uma tecnologia que está em desenvolvimento com potencial de novos avanços tecnológicos.

Com a crescente preocupação nutricional e ecológica vivida na atualidade, três principais nichos tecnológicos podem ser explorados:

a) Tecnologias que melhorem ou modifiquem as etapas de refino de maneira a gerar um produto final de melhor qualidade nutricional;

b) Tecnologias que envolvam um produto final com propriedades medicinais; $e$

c) Tecnologias que alinhem processos de produção de óleos comestíveis à diversidade do ecossistema local, aproveitando-se do potencial de matérias-primas regionais para obtenção desses óleos de forma econômica e sustentável.

Os processos mecânicos que conservam o valor nutricional são ainda menos utilizados por serem menos eficazes que processos químicos. Por outro lado, o processamento químico ainda possui gargalos, como a etapa de extração do óleo bruto, o tempo de processamento e a etapa de neutralização por tratamento alcalino, o que pode prejudicar o valor nutricional do produto final, eliminando componentes valiosos que foram ainda pouco explorados.

A indústria de produção de óleos comestíveis é um setor bem consolidado, mas pela necessidade de se adequar às tendências mundiais de mercado e de atender às políticas internacionais de proteção, acompanhada da crescente preocupação em se consumir produtos alimentícios obtidos por mecanismos mais sustentáveis, espera-se que as empresas sejam as principais investidoras nesse tipo de tecnologia nos próximos anos, impulsionando um aumento nas contribuições tecnológicas sobre refino de óleos comestíveis.

\section{Referências}

ADHAMI, K. et al. A novel process for simultaneous degumming and deacidification of Soybean, Canola and Sunflower oils by tetrabutylphosphonium phosphate ionic liquid. Journal of Industrial and Engineering Chemistry, [s.l.], v. 76, p. 245-250, ago. 2019.

ALIPOUR, S. et al. $\beta$-carotene production from soap stock by loofa-immobilized Rhodotorula rubra in an airlift photobioreactor. Process Biochemistry, [s.l.], v. 54, p. 9-19, mar. 2017.

BARBOSA, L. C. F. et al. Inventores. PETROBRÁS. Titular. Processo para recuperação avançada de petróleo pelo uso de solução de co-produtos da fabricação do biodiesel. Patente BR n. PI 0901604-0 A2. 2009.

CARGILL, 2020. Disponível em: http://cargill.com/about/cargill-history. Acesso em: 24 jul. 2020.

DUIJN, G. Fate of contaminants during the refining process of vegetable oils and fats: a calculation model. European Journal of Lipid Science and Technology, [s.l.], v. 118, n. 3, p. 353-360, mar. 2016.

FALK, B. Inventor. CARGILL INC. Titular. Palm oil without unwanted contaminants. Patente WO2019US16998. 2018a. 
FALK, B. Inventor. CARGILL INC. Titular. Liquid oils without unwanted contaminants. WO2019US16994. 2018b.

FAZLI, Y. et al. Soap stock separation process. Asian Journal of Chemistry, [s.l.], v. 25, n. 4, p. 2333-2334, mar. 2013.

FREEMAN et al. Inventores. CARGILL INC. Titular. Nut-like food compositions. Patente WO2002US16189. 2001.

FU, D. et al. Research progress and strategies for multifunctional rapeseed: a case study of China. Journal of Integrative Agriculture, [s.l.], v. 216, p. 1.673-1.684, 2016.

FUKUHARA, S. et al. Inventores. KAO CORPORATION. Titular Manufacturing method of purified fish oil. Patente JP20130248898. 2013.

HANBEI, M. Inventor. HENAN FENGXIANG PEONY TECNOLOGY CO LTD. Titular. Peony seed formula oil. Patente CN201910737518. 2019.

HIDAKA, I.; TSUCHIYA, N. Inventores. NISSHIN OILLIO GROUP LTD. Titular. Preparation of Rice bran oil. Patente JP19980293157. 1998.

HILTEN, R. et al. Production of aromatic green gasoline additives via catalytic pyrolysis of acidulated peanut oil soap stock. Bioresource Technology, [s.l.], v. 102, p. 8.288-8.294, set. 2011.

HIROSHI, H. Inventor. NISSHIN OILLIO GROUP LTD. Titular. Method for producing refined palm-based fat. Patente JP20140064425. 2014.

HIROSHI, H. Inventor. NISSHIN OILLIO GROUP LTD. Titular. Manufacturing method of refined oil and fat and manufacturing management method of refined oil and fat. Patente JP20150007614. 2015.

IWASAWA et al. Inventores. NISSHIN OILLIO GROUP LTD. Titular. Method for producing edible oil, and the edible oil produced by the method. Patente JP20090049912. 2009.

KAO. 2020. Disponível em: http://chemical.kao.com/global/business/. Acesso em: 24 jul. 2020.

KRUIDENBERG, M. B.; FALK, B. Inventores. CARGILL INC. Titular. Oil compositions. Patente CN201310324096. 2011.

KUNO, N.; SHINOHARA, T. Inventores. NISSHIN OILLIO GROUP LTD. Titular. Process for producing fat compositions containing oleanolic acid and/or maslinic acid. Patente JP20030532614. 2001.

LEE, Z. S. et al. Treatment Technologies of palm oil Mill effluent (POME) and olive mill wastewater (OMW): A brief review. Environmental Technology and Innovation, [s.l.], v. 15, n. 100377, ago. 2019.

LI, S. et al. Systematic qualitative and quantitative assessment of fatty acids in the seeds of 60 tree peony (Paeonia section Moutan DC.) cultivars by GC-MS. Food Chemistry, [s.l.], v. 173, p. 133-140, 2015.

LIU, D. et al. Inventores. ANLU TIANXING FOOD \& OIL PROC MACHINERY EQUIPMENT. Titular. Production method for preparing and refining oil from tea seeds or camélia seeds. Patente CN201410299111. 2014. 
LIU, L.; LAMPERT, D. S. Inventores. CARGILL INC. Titular. Partial interesterification of triacylglycerols. Patente US19970932755. 1997.

MAKI et al. Inventores. NISSHIN OILLIO GROUP LTD. Titular. Palm oil, deodorized distillates and manufacturing methods therefor. Patente JP20100535789. 2008.

MARQUES, L. S. et al. Mapeamento patentário de recuperação avançada de petróleo (EOR) com aditivos poliméricos/biopoliméricos e surfactantes. Cadernos de Prospecção, Salvador, v. 7, n. 2, p. 198-207, abr.-jun. 2014.

MASAYUKI, I.; HIROYUKI, K. Inventores. NISSHIN OILLIO GROUP LTD. Titular. Manufacturing system of edible oil, and manufacturing method of edible oil. Patente JP20170190901. 2017.

MASAYUKI, I. Inventor. NISSHIN OILLIO GROUP LTD. Titular. Manufacturing system of edible oil, and manufacturing method of edible oil. Patente JP20170190900. 2017.

MELO, E. et al. First study on the oxidative stability and elemental analysis of babassu (Attalea speciosa) edible oil produced in Brazil using a domestic extraction machine. Molecules, [s.l.], v. 24. n. 4.235, dez. 2019.

MINORU, K.; TOSHITERU, K. Inventores. KAO CORPORATION. Titular. Method for manufacturing refined fats and oils. Patente KR20137033215. 2011.

NI, C. et al. Phase transformation of thermoresponsive surfactant triggered by its concentration and temperature. Journal of Petroleum Science and Engineering, [s.l.], v. 193, n. 107410, out. 2020.

NIKKEI ASIAN REVIEW. 2020. Disponível em: http://asia.nikkei.com/Companies/The-Nisshin-OilliOGroup-Ltd. Acesso em: 23 jul. 2020.

NUCCI, B. et al. Improving the environmental performance of vegetable oil processing through LCA. Journal of Cleaner Production, [s.l.], v. 64, p. 310-322, 2014.

PANTOJA, S. S. et al. High-quality biodiesel production from buriti (Mauritia flexuosa) oil soapstock. Molecules, [s.l.], v. 24, n. 94, jan. 2019.

POYARKOVA, T. N. et al. Dependence of the stability of a soap stock emulsion on the combined effect of sodium chloride and nonionogenic surfactant additives. Russian Journal of Applied Chemistry, [s.l.], v. 86, n. 2, p. 200-205, fev. 2013.

POYARKOVA, T. N. et al. Effect of inorganic electrolytes and noionogenic surfactants on the stability of soap stock emulsion. Russian Journal of Applied Chemistry, [s.l.], v. 85, n. 4, p. 651-655, abr. 2012.

RAMAN, K. Inventor. DSM IP ASSETS BV. Titular. Microbial oils enriched in polyunsaturated fatty acids. Patente EP20120815593. 2011.

RODRIGUES, P. D.; QUINTELLA, C. M. Prospecção tecnológica de patentes sobre a obtenção de ácidos graxos a partir da borra de refino do tratamento de neutralização de óleos brutos (soap stock). Cadernos de Prospecção, Salvador, v. 10, n. 3, p. 563-577, jul.-set. 2017.

SAMPAIO, C. J. S. et al. Estudos de prospecção sobre matriz polimérica para imobilização de bactérias e uso em biorremediação. Cadernos de Prospecção, Salvador, v. 12, n. 3, p. 639-650, 2019. 
SANDBERG, S.; THURSTAD, O. Inventores. BASF AG. Titular. Removal of undesired components from oil compositions. Patente CN201910956575. 2012.

SANTOS, S. C. et al. Mapeamento tecnológico de processos microbianos aplicados na biorremediação de metais pesados. Cadernos de Prospecção, Salvador, v. 11, n. 5, p. 1.740$1.751,2018$.

SHAHBANDEH, M. Vegetable oils: global consumption by oil type 2013/14 to 2019/2020. Statista, 31 jan. 2020. Disponível em: https://www.statista.com/statistics/263937/vegetable-oils-globalconsumption/. Acesso em: 8 maio 2020.

SI, Z.; SCOTT, S. As mudanças alimentares da China e suas implicações globais. Diálogo Chino, Londres, 23 jan. 2019. Disponível em: https://dialogochino.net/pt-br/agricultura-pt-br/21163-asmudancas-alimentares-da-china-e-suas-implicacoes-globais/. Acesso em: 24 set. 2020.

SONG, D.; FANG, H. Inventores. BENGBU JIANGHUAI GRAIN \& OIL. Titular. Rapeseed oil pressing method capable of lowering water content. Patente CN201710412975. 2017.

SONG, Y. Inventor e titular. Preparation method of cyperus esculentus oil. Patente CN201910885698. 2019.

SUN, C. et al. Inventores. ACADEMY STATE ADMINISTRATION OF GRAIN. Titular. Method for industrially removing zearalenone out of maize germ. Patente CN20130162748. 2013.

TAVAKOLI, A. et al. Optimization of high voltage electric field as a novel non-thermal method of sunflower oil neutralization. Separation and Purification Technology, [s.l.], v. 211, p. 430-437, mar. 2019.

TETSUYA et al. Inventores. KAO CORPORATION. Titular. Method for producing triterpene alcohol-containing fat and oil. Patente JP20130248899. 2013.

TRAITLER, H.; WINTER, H. Inventores. NESTLÉ. Titular. Food compositions containing fatty substances and a process for the preparation thereof. Patente ZA19830002492. 1982.

WALKER, C. The effects of an american diet on health. UAB's Undergraduate Research Journal, [s.l.], v. 9, 2015.

WANG, Y.; ZHOU, S. Inventores. FENGYI SHANGAI BIOTECHNOLOGY. Titular. Sunflower seed oil retaining more active nutritional components. Patente CN201611176007. 2016.

YAMAUCHI et al. Inventores. NISSHIN OILLIO GROUP LTD. Titular. Conjugated fatty acid containing monoglycerides and process for producing them. Patente DK20050022008T. 2001.

YOSHINORI, A.; TOSHITERU, K. Inventores. KAO CORPORATION. Titular. Method fpr producing fat and oil including reduced amount of free fatty acid. Patente JP20040271690. 2004.

ZEYANG, X. Inventor. GUIZHOU LONGLI ZEYANG ECOLOGICAL TECH PLANTATION AND CULTIVATION CO LTD. Titular. Extraction method of peony seed oil. Patente CN201610945945. 2016.

ZIKUAN, M. et al. Inventores. SICHUAN FOOD RESEARCH AND DESIGN INSTITUTE OF FOOD FERMENTATION INDUSTRY. Titular. Processing method and product of selenium-rich rapeseed oil. Patente CN20191131277. 2019. 


\section{Sobre as Autoras}

\section{Yara Simone Chaves Sousa}

E-mail: yarasimone@hotmail.com

ORCID: https://orcid.org/0000-0001-8361-5040

Mestra em Química pela Universidade Federal da Bahia em 2014. http://lattes.cnpq.br/1506670550963153. (Bolsista CAPES n. 88882.452749/2019-01).

Endereço profissional: Universidade Federal da Bahia, Instituto de Química, Departamento de Química Geral e Inorgânica, Campus de Ondina, Ondina, Salvador, BA. CEP: 40170-270.

\section{Pamela Dias Rodrigues}

E-mail: pamelarodrigues.ufba@gmail.com ORCID: https://orcid.org/0000-0003-2338-5057

Doutora em Química pela Universidade Federal da Bahia em 2018. http://lattes.cnpq.br/4554329622469373.

Endereço profissional: Universidade Federal da Bahia, Instituto de Química, Departamento de Química Geral e Inorgânica, Campus de Ondina, Ondina, Salvador, BA. CEP: 40170-290.

\section{Cristina M. Quintella}

E-mail: cris5000tina@gmail.com ORCID: http://orcid.org/0000-0002-3827-7625

Doutora em Ciências Moleculares pela University of Sussex (UK) em 1993. http://lattes.cnpq.br/78977798819494573. Endereço profissional: Universidade Federal da Bahia, Instituto de Química, Departamento de Química Geral e Inorgânica, Campus de Ondina, Ondina, Salvador, BA. CEP: 40170-290. 